\title{
Changes in the Polysomnographic Measures in Patients of Chronic Insomnia on Drug Therapy Vs Mindful Awareness
}

\author{
Shweta Kanchan ${ }^{1}$, B.D Singh ${ }^{2}$, Gautam Swaroop ${ }^{3}$, Gynendra Kumar ${ }^{4}$ \\ ${ }^{1}$ Post MD PhD Scholar Department of Physiology KGMC Lucknow, ${ }^{2} H O D$ Department of Physiology MLB \\ Medical College Jhansi, ${ }^{3}$ Consultant Cardiologist Sahara Hospital Lucknow ${ }^{4} H O D$ Department of Psychiatry MLB \\ Medical College Jhansi
}

\begin{abstract}
Background:- Modern day $24 * 7$ lifestyle is witnessing an increase in people with insomnia like never before. The deep and restoring sleep like a baby seems to have vanished from our hectic lifestyles, and sleep disorders like insomnia have crept in involving people across all ages and sections of society. In the given scenario it remains a matter of concern that most primary physicians are not trained in the various modalities which could be used for the treatment of insomnia. In the absence of required knowledge in insomnia management hypnotic medication remain the preferred treatment for insomnia. The present study was undertaken with a view to study the effectiveness of mindfulness based relaxation therapy vs hypnotic medication for the treatment of insomnia.

Methods :- A cross sectional study was conducted on 100 patients suffering from insomnia in two groups, pharmacotherapy (PCT) group and Mindfulness based stress reduction (MBSR) group. MBSR a program of mindfulness meditation training consisting of one hour long class daily which includes training in progressive muscular exercises, breathing exercises, standing, sitting and walking meditations. Home practice expectations were 30 minutes of medication per day and following an attitude of mindfulness through out the day during the four week follow.

Result :- The study showed comparable results on all sleep parameters for both groups. There was a significant increase in total sleep time in both groups, the increase was more with drug group compare to the MBSR group.

Conclusion :- While the time commitment associated with participating in and practicing a behavioral intervention such as MBSR is more than with medication, our results suggest that this is not a deterrent to most of our participants. Given patient preferences, the side effects of pharmacotherapy, evidence of the efficacy of MBSR and the potential positive benefits of meditation that go beyond management of insomnia symptoms.
\end{abstract}

Keywords:- Insomnia, Pharmacotherapy, Relaxation Therapy, Mindful awareness.

\section{Introduction}

\section{Sleep and Insomnia}

Sleep has been defined behaviorally as a

\section{Correspondence Info:}

\section{Dr. B.D.Singh}

Associate Professor \& HOD Deptt. of Physiology,

MLB Medical College Jhansi

E-mail Id :- bdssagar@gmail.com reversible state of perceptual disengagement from and unresponsiveness to the environment. Sleep is a complex state in which changes occur in physiologic and behavioral processes just like with wakefulness. Sleep is physiologic, necessary, temporary, reversible, and cyclic. To sustain optimal alertness throughout the day, the requirement varies across individuals, with the mean being 7 to 8 hours for adult humans.

The term insomnia refers to a condition characterized by difficulties initiating and/or maintaining sleep, 
accompanied by clinically significant daytime sleepiness or distress related to the ongoing sleep difficulties Given this observation, it is best to use the term comorbid insomnia when prominent, clinically significant insomnia symptoms are observed concurrent to another medical, psychiatric, or sleep disorder .

The modern era of hypnotic pharmacology began in the 1960s with the introduction of the benzodiazepines ,or diazepam-like compounds, which dominated until the development of the newer, nonbenzodiazepine agents in the 1990s All of the current FDA-approved agents - with the exception of ramelteon and doxepinact by modulating the function of the $\gamma$-amino butyric acid (GABA) ${ }^{1}$.

\section{Mindfullness based stress reduction}

The Mindfullness based stress reduction ,MBSR facilitates adaptation to the stresses of living ${ }^{2}$. The MBSR program teaches participants to learn how to focus their attention through a variety of meditative techniques. Participants are trained to perceive their immediate emotional and physical state, including pain or discomfort, and to let thoughts come and go in awareness with no attempt to change, suppress or elaborate on thoughts. Through mindfulness training, participants learn to view their thoughts as mental events and not facts. In this way, participants become exposed to the positive and negative content of their thoughts, and do not get absorbed in thought, caught up in planning for the future or worrying about the past. By "breaking up" cycles of rumination and worry, mindfulness is hypothesized to reduce "verbal over-regulation" and facilitate the dis-engagement necessary to fall asleep ${ }^{3}$.

\section{Aims \& Objective}

The aim of this study was to compare the effectiveness of mindfullness based stress relaxation therapy MBSR vs commonly taken medicine zolpidem in altering the sleep parameters in whole night polysomnographic studies in patients of chronic insomnia

\section{Inclusion Criteria}

Patients were recruited between July, 2012 and September 2012, by clinician referral. Age 18 to 65 Ability to read and speak hindi,
Diagnosis of primary chronic insomnia. Chronic insomnia was defined as difficulty initiating or maintaining sleep despite adequate opportunity for sleep, with related daytime dysfunction on 3 or more nights a week for the past 6 months or )longer, consistent with the DSM-IV-TR and International Classification of Sleep Disorders (ICSD-3) ${ }^{4}$.

\section{Exclusion Criteria:}

Persons with medical conditions, mental disorders, or different sleep disorders suspected of being directly related to the insomnia, those taking medications affecting sleep were excluded.

\section{Material and Method}

MBSR, a program of mindfulness meditation training consisting of one hour long class daily for 5 days which included training in progressive muscular exercises, breathing exercises, standing, sitting and walking meditations, Home practice expectations were 30 minutes of meditation per day and following an attitude of mindfulness throughout the day during the 4 week follow-up; Our pharmacotherapy group was modeled on clinical practice. It is standard and common practice to prescribe on a nightly basis for a number of months during the initial management of chronic primary insomnia.

The PCT treatment consisted of $.5 \mathrm{mg}$ of zolpidem nightly for 4 weeks. Patients initially met in a small group with the sleep physician who gave instructions for properly taking medication and explained potential side effect . A 10-minute sleep hygiene presentation was included in both interventions. Both groups were asked to practice sleep restriction and not indulge in afternoon nap, no caffeinated drink after 3:pm The study was conducted on 100 patients suffering from insomnia who presented to the OPD of M L B Medical College, Jhansi, with the chief complain of insomnia. They were divided into two groups one group was given $5 \mathrm{mg}$ zolpidem every night, inetial polysomnographic studies were conducted followed by PSG every week for 4 weeks to monitor the changes in sleep parameters.). Screening protocol applied for diagnostic criteria for primary insomnia included a structured psychiatric interview (SCID-IV), completion of a screening sleep diary, and a history and physical examination conducted 
by a physician with training in sleep medicine group.

\section{Observation}

Table 1 Shows- Result of drug versus mindfullnress on sleep study parameters

\begin{tabular}{|c|c|c|c|c|c|}
\hline \multicolumn{6}{|c|}{ Paired Samples Statistics } \\
\hline \multicolumn{2}{|c|}{$\begin{array}{l}\text { TOTAL SLEEP } \\
\text { TIME }\end{array}$} & \multirow{2}{*}{$\begin{array}{l}\text { Mean } \\
315.10\end{array}$} & \multirow{2}{*}{$\begin{array}{l}\mathbf{N} \\
30\end{array}$} & \multirow{2}{*}{$\begin{array}{l}\text { Std. Deviation } \\
10.842\end{array}$} & \multirow{2}{*}{$\begin{array}{l}\text { Std. Error Mean } \\
1.979\end{array}$} \\
\hline Pair 1 & bd & & & & \\
\hline & $\mathrm{ad}$ & 345.60 & 30 & 10.301 & 1.881 \\
\hline \multirow{2}{*}{ Pair 2} & bt & 301.13 & 30 & 36.757 & 6.711 \\
\hline & at & 322.03 & 30 & 41.018 & 7.489 \\
\hline \multicolumn{6}{|c|}{ SLEEP LATENCY } \\
\hline \multirow{2}{*}{ Pair 1} & $\mathrm{Bd}$ & 49.93 & 30 & 1.982 & .362 \\
\hline & Ad & 29.30 & 30 & 2.756 & .503 \\
\hline \multirow[t]{2}{*}{ Pair 2} & $\mathrm{Bt}$ & 50.30 & 30 & 2.037 & .372 \\
\hline & At & 25.43 & 30 & 1.695 & .310 \\
\hline \multicolumn{6}{|c|}{ SLEEP EFFICIENCY } \\
\hline \multirow[t]{2}{*}{ Pair 1} & $\mathrm{Bd}$ & 77.70 & 30 & 3.395 & .620 \\
\hline & $\mathrm{Ad}$ & 85.73 & 30 & 3.503 & .640 \\
\hline \multirow[t]{2}{*}{ Pair 2} & $\mathrm{Bt}$ & 78.57 & 30 & 2.661 & .486 \\
\hline & At & 89.43 & 30 & 2.176 & .397 \\
\hline \multicolumn{6}{|c|}{ REM LATENCY } \\
\hline \multirow[t]{2}{*}{ Pair 1} & bd & 106.97 & 30 & 3.978 & .726 \\
\hline & $\mathrm{ad}$ & 135.70 & 30 & 4.027 & .735 \\
\hline \multirow[t]{2}{*}{ Pair 2} & bt & 105.33 & 30 & 3.536 & .646 \\
\hline & at & 130.10 & 30 & 3.689 & .674 \\
\hline \multicolumn{6}{|c|}{ WAKE AFTER SLEEP ONSET } \\
\hline \multirow[t]{2}{*}{ Pair 1} & bd & 45.83 & 30 & 3.455 & .631 \\
\hline & $\mathrm{ad}$ & 62.33 & 30 & 3.294 & .601 \\
\hline \multirow[t]{2}{*}{ Pair 2} & bt & 46.67 & 30 & 4.302 & .785 \\
\hline & at & 62.27 & 30 & 3.084 & .563 \\
\hline \multicolumn{6}{|c|}{ TIME IN REM SLEEP } \\
\hline \multirow[t]{2}{*}{ Pair 1} & bd & 49.43 & 30 & 3.137 & .573 \\
\hline & $\mathrm{ad}$ & 48.00 & 30 & 2.971 & .542 \\
\hline \multirow[t]{2}{*}{ Pair 2} & bt & 50.57 & 30 & 3.126 & .571 \\
\hline & at & 48.80 & 30 & 3.134 & .572 \\
\hline \multicolumn{6}{|c|}{ AROUSAL } \\
\hline \multirow[t]{2}{*}{ Pair 1} & bd & 8.00 & 30 & .830 & .152 \\
\hline & $\mathrm{ad}$ & 6.63 & 30 & .718 & .131 \\
\hline \multirow[t]{2}{*}{ Pair 2} & $\mathrm{bt}$ & 8.00 & 30 & .871 & .159 \\
\hline & at & 4.17 & 30 & .699 & .128 \\
\hline
\end{tabular}

bd-before starting drug therapy, ad- after starting the drug therapy, bt-before the mindfulness therapy, at- after mindfulness therapy 
Table-2 shows a significant difference in all parameters(pvalue $<.05$ ) both after drug and mindfulness therapy

\begin{tabular}{|c|c|c|c|c|c|c|c|c|c|}
\hline \multicolumn{10}{|c|}{ Paired Samples Test } \\
\hline \multirow{3}{*}{\multicolumn{2}{|c|}{$\begin{array}{l}\text { TOTAL SLEEP } \\
\text { TIME }\end{array}$}} & \multicolumn{5}{|c|}{ Paired Differences } & \multirow{4}{*}{$\begin{array}{l}\mathbf{t} \\
-46.549\end{array}$} & \multirow{4}{*}{$\begin{array}{r}\text { df } \\
\\
29\end{array}$} & \multirow{4}{*}{$\begin{array}{l}\begin{array}{l}\text { Sig. } \\
\text { (2-tailed) }\end{array} \\
.000\end{array}$} \\
\hline & & \multirow{3}{*}{$\begin{array}{l}\text { Mean } \\
-30.500\end{array}$} & \multirow{3}{*}{$\begin{array}{l}\text { Std. } \\
\text { Deviation }\end{array}$} & \multirow{3}{*}{$\begin{array}{l}\text { Std. } \\
\text { Error } \\
\text { Mean }\end{array}$} & \multicolumn{2}{|c|}{$\begin{array}{l}\text { 95\% Confidence } \\
\text { Interval of the } \\
\text { Difference }\end{array}$} & & & \\
\hline & & & & & Lower & Upper & & & \\
\hline Pair 1 & bd - ad & & & & -31.840 & -29.160 & & & \\
\hline Pair 2 & bt - at & -20.900 & 18.159 & 3.315 & -27.681 & -14.119 & -6.304 & 29 & .000 \\
\hline \multicolumn{10}{|c|}{ SLEEP LATENCY } \\
\hline Pair 1 & bd - ad & 20.633 & 1.938 & .354 & 19.910 & 21.357 & 58.302 & 29 & .000 \\
\hline Pair 2 & bt- at & 24.867 & 1.042 & .190 & 24.478 & 25.256 & 130.753 & 29 & .000 \\
\hline \multicolumn{10}{|c|}{ SLEEP EFFICIENCY } \\
\hline Pair 1 & bd - ad & -8.033 & .890 & .162 & -8.366 & -7.701 & -49.443 & 29 & .000 \\
\hline Pair 2 & bt - at & -10.867 & 1.502 & .274 & -11.428 & -10.306 & -39.614 & 29 & .000 \\
\hline \multicolumn{10}{|c|}{ REM LATENCY } \\
\hline Pair 1 & bd - ad & -28.733 & 1.363 & .249 & -29.242 & -28.224 & -115.474 & 29 & .000 \\
\hline Pair 2 & bt - at & -24.767 & 1.775 & .324 & -25.429 & -24.104 & -76.425 & 29 & .000 \\
\hline \multicolumn{10}{|c|}{ WAKE AFTER SLEEP ONSET } \\
\hline Pair 1 & bd - ad & -16.500 & 3.330 & .608 & -17.743 & -15.257 & -27.143 & 29 & .000 \\
\hline Pair 2 & bt - at & -15.600 & 2.711 & .495 & -16.612 & -14.588 & -31.513 & 29 & .000 \\
\hline \multicolumn{10}{|c|}{ TIME IN REM SLEEP } \\
\hline Pair 1 & bd - ad & 1.433 & .728 & .133 & 1.162 & 1.705 & 10.785 & 29 & .000 \\
\hline Pair 2 & bt - at & 1.767 & .679 & .124 & 1.513 & 2.020 & 14.253 & 29 & .000 \\
\hline \multicolumn{10}{|c|}{ AROUSAL } \\
\hline Pair 1 & bd - ad & 1.367 & .490 & .089 & 1.184 & 1.550 & 15.272 & 29 & .000 \\
\hline Pair 2 & bt - at & 3.833 & .648 & .118 & 3.591 & 4.075 & 32.415 & 29 & .000 \\
\hline
\end{tabular}

bd-before starting drug therapy, ad- after starting the drug therapy, bt-before the mindfulness therapy, atafter mindfulness therapy 


\section{Observation}

The study showed comparable results on all sleep parameters for the two groups.There was a significant increase in total sleep time in both groups, the increase was more with the drug group compared to the MBSR group. The decrease in sleep latency was almost equal in both groups being only marginally more in the drug group (20.63 $\mathrm{min}$ vs $24.8 \mathrm{~min}$ ). The improvement in sleep effciency was almost similar in both groups, it was increased slightly more in the MBSR group 89.73 vs 85.43 .The increase in REM latency was more in the drug group $28.73 \mathrm{~min}$ vs $24.76 \mathrm{~min}$.The changes in wake aGer sleep onset time was almost equal in both groups 16.3 vs $15.5 \mathrm{~min}$. Time in REM was decreased to a similar extent in both groups $1.43 \mathrm{~min}$ vs $1.76 \mathrm{~min}$. The number of arousals decreased more with MBSR compared to drug 3.83 vs $1.36 \mathrm{~min}$.

\section{Discussion}

This study provides initial evidence for the efficacy of a complementary and alternative treatment modality, MBSR, as a viable treatment for chronic insomnia as measured by changes in polysomnographic parameters Our results suggest that MBSR, when combined with a brief sleep hygiene presentation, is able to achieve reductions in improvements in sleep quality comparable to regular use of an FDA-approved sedative hypnotic..

Patients who completed 5 or more MBSR classes reported sleep changes that were large and clinically meaningful - total sleep time increased by over 30 minutes, sleep onset latency reduced by over 20 minutes and sleep efficiency increased to $88.5 \%$. Moreover the patients randomized to MBSR met stringent criteria for recovery from insomnia at the end of the study, and average treatment satisfaction scores were high. Whereas patients in the PCT arm obtained similar benefits to sleep outcomes, their treatment satisfactions scores were not high,

Impacts found following MBSR compare favorably to outcomes reported from trials of cognitive-behavioral therapy (CBT) for patients with chronic or persistent insomnia. Morin et al. ${ }^{5}$ recently reported the results of a trial of where adults with persistent insomnia were randomized to 6 weeks of group CBT. These patients improved from an average ISI score of 17.26 at baseline to an average of 8.11 at 6 month follow-up; sleep efficiency measured by diary improved from $69 \%$ to $82.4 \%$, and large improvements in sleep onset latency and time awake after sleep onset were also found. Our finding of durable improvements to sleep outcomes from MBSR is consistent with results reported by Edinger et al. ${ }^{6}$ from their seminal trial of CBT in primary insomnia.

Our findings build upon positive results from several longitudinal studies of mindfulness-based treatment approaches with insomnia patients. Three uncontrolled studies with a total of 56 patients and one waitlist-controlled trial with 52 patients reported reductions in insomnia symptoms and improvements on other sleep outcomes in patients with mood and/or anxiety disorders following a MBCT. Ree and Craigie included the ISI in a study of MBCT for psychiatric outpatients with insomnia ${ }^{7}$., and reported significant improvement (ISI, $d=.84$ ) for 23 patients following the program, and benefits maintained at 3 month followup. Heidenrich et $\mathrm{al}^{8}{ }^{8}$ reported that 14 patients with refractory chronic insomnia co-morbid with other mental disorders showed pre- to post-MBCT improvements in total sleep time and sleep latency measured by sleep diary, and a decline in dysfunctional thoughts about insomnia. Yook et al. ${ }^{9}$ reported PSQI scores were significantly decreased among 19 patients with anxiety disorders and insomnia after an 8-week MBCT program. Britton et al. ${ }^{10}$ studied the sleep outcomes of 7 women with insomnia following an abbreviated MBSR program and found that WASO measured by sleep diary was reduced. In a subsequent waitlist-controlled trial, Britton et al.5 enrolled adults with insomnia co-morbid with depression, and randomized them to an 8-week MBCT program or a waitlist. Compared to controls $(n=17)$, MBCT participants' sleep diary reports $(n=25)$ indicated significantly shorter WASO, and trends for decreased SOL and decreased awakenings, adjusted for use of antidepressants. These studies, all of which found reductions in one or more measures of mood or cognition (depression, anxiety, worry or rumination) as well as sleep improvements, complement the growing literature on the health benefits of mindfulness training with MBSR These findings, in conjunction with the results of the present study, suggest that mindfulness training has potentially broad application for improving insomnia and closely associated problems that may perpetuate insomnia - symptoms of anxiety and depression. 
Strengths of our study include a rigorous screening process to eliminate individuals likely to have insomnia due to another underlying disorder. We tried to stick with MBSR along with training in basic sleep hygine so that the findings could be tested in future studies and be generalised.. Limitations included lack of additional control groups, such as a medication placebo and a behavioral attention control, to exclude the possibility that non-specific factors such as expectancy, attention, or regression to the mean might account for the positive effects found. Other limitations include the homogeneity of participants in terms of belonging to same geographical area and race. Another limitation was a lack of follow up which would help us know if the changes in sleep parameters were sustained after few months of inetiating therapy .Our study focused on changes in polygraphic measures only, a more comprehensive study including changes in patient sleep diaries, actigraphy and daytime impairment due to insomnia.

\section{Conclusion}

While the time commitment associated with participating in and practicing a behavioral intervention such as MBSR is more than with medication, our results suggest that this is not a deterrent to most of our participants. Given patient preferences, the side effects of pharmacotherapy, evidence of the efficacy of MBSR and the potential positive benefits of meditation that go beyond management of insomnia symptoms, it is important that health care providers be aware of the range of non-pharmacologic therapy approaches and that clinicians offer patients options that include MBSR. Future studies of MBSR for insomnia should employ larger sample sizes, and longer follow up to assess the durability of treatment interventions, and include design features that could reveal mechanisms of action and deduce the most effective components of this intervention.

Ethical Clearance: Present study was approved by institutional and review committee. MLB Medical College Jhansi, UP India.

Conflict of Interest - Nil

Source of Funding- Self

\section{References}

1. Janette D. Lie, PharmD, BCACP; Kristie N. Tu, PharmD, BCPS, CGP; Diana D. Shen, PharmD Candidate; and Bonnie M. Wong, PharmD Candidate Pharmacological Treatment of Insomnia pharmacy and therapeutics 2015 Nov; 40(11): 759768,771 .

2. Morin CM, LeBlanc M, Daley M, Gregoire JP, Merette C. Epidemiology of insomnia: prevalence, self-help treatments, consultations, and determinants of help-seeking behaviors. Sleep Medicine. 2006;7:123-30.

3. Winbush NY, Gross CR, Kreitzer MJ. The e $\square$ ects of mindfulness-based stress reduction on sleep disturbance: A systematic review. EXPLORE: The Journal of Science \& Healing. 2007;3:585-91.

4. American Academy of Sleep Medicine . International Classification of Sleep Disorders: 2nd Ed Diagnostic and Coding Manual: American Academy of Sleep Medicine. 2005.

5. Morin CM, LeBlanc M, Daley M, Gregoire JP, Merette C. Epidemiology of insomnia: prevalence, self-help treatments, consultations, and determinants of help-seeking behaviors. Sleep Medicine. 2006;7:123-30.

6. Edinger JD, Wohlgemuth WK, Radtke RA, Marsh GR, Quillan RE. Cognitive behavioral therapy for treatment of chronic primary insomnia: A randomized controlled trial. JAMA. 2001;285:1856-64.

7. Bastien $\mathrm{CH}$, Vallieres A, Morin CM. Validation of the Insomnia Severity Index as an outcome measure for insomnia research. Sleep Medicine. 2001;2:297-307

8. Heidenreich T, Tuin I, Pflug B, Michal M, Michalak J. Letter to the editor: Mindfulness-based cognitive therapy for persistent insomnia: A pilot study Psychother Psychosom. Vol. 752006.

9. Yook K, Lee SH, Ryu M, et al. Usefulness of mindfulness-based cognitive therapy for treating insomnia in patients with anxiety disorders. A pilot 
study The Journal of Nervous and Mental Disease. 2008;196:501-3.]

10. Grossman P, Niemann L, Schmidt S, Walach H.
Mindfulness-based stress reduction and health benefits. A meta-analysis. J Psychosom Res. 2004;57:35-43. ] 